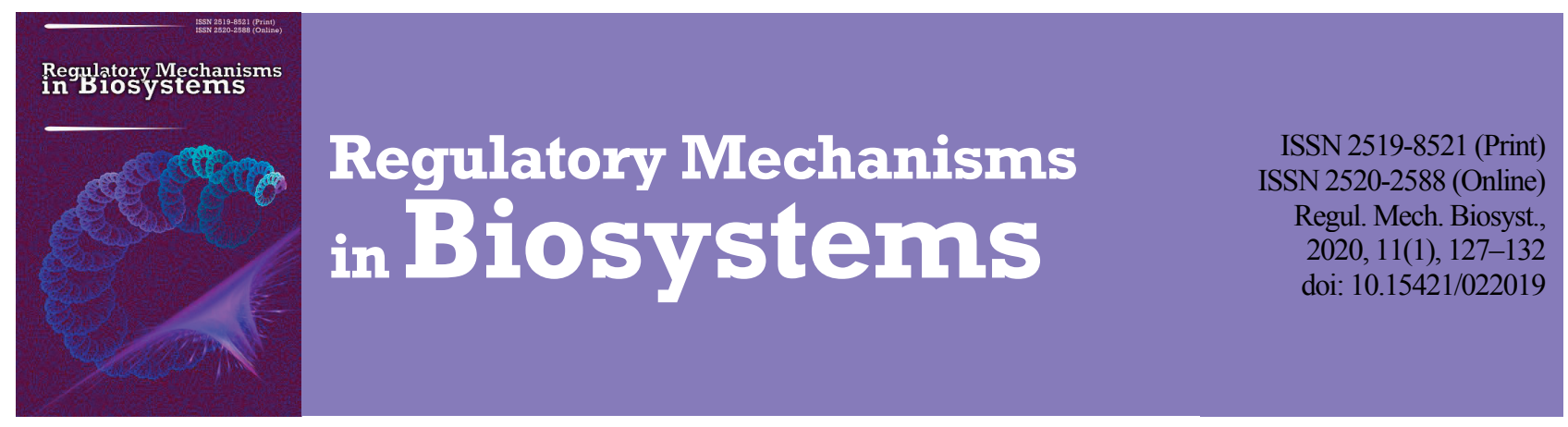

\title{
In vitro synergy testing of prodigiosin in combination with inhibitors of cell wall synthesis against Mycobacterium smegmatis
}

\author{
D. A. Ivanchenko, L. M. Hrytsenko \\ Bogomolets National Medical University, Kyiv, Ukraine
}

Article info

Received 06.01.2020

Received in revised form 02.02 .2020

Accepted 03.02 .2020

Bogomolets National Medical University,

Shevchenka boulevard, 13 ,

Kyiv, 01601, Ukraine.

Tel.: +38-097-265-23-25.

E-mail:

larysa.hrytsenko@gmail.com

Ivanchenko, D. A., \& Hrytsenko, L. M. (2020). In vitro synergy testing of prodigiosin in combination with inhibitors of cell wall synthesis against Mycobacterium smegmatis. Regulatory Mechanisms in Biosystems, 11(1), 127-132. doi:10.15421/022019

The cell wall is not a target of currently used therapeutics as Mycobacterium are considered naturally resistant to most $\beta$-lactam antibiotics. Therefore, combinations of conventional antibiotics with antibiotic activity-enhancing compounds offer a productive treatment strategy and address the widespread emergence of antibiotic-resistant strains. The first area of research was the study of a comparative analysis of disk diffusion testing and the broth dilution method for evaluating the susceptibility of $M$. smegmatis to antimicrobial agents. A comparative analysis of the susceptibility to antimicrobial agents alone showed that $M$. smegmatis was the most susceptible to ceftriaxone and kanamycin, and moderately sensitive to vancomycin and prodigiosin. Compared to the susceptibility of the antibacterial combinations, the isolate was not susceptible to antibacterial combinations with prodigiosin in disk diffusion testing. The second area of research was the study of the synergic activity of prodigiosin of $S$. marcescens and inhibitors of cell wall synthesis manifested by their simultaneous effect on $M$. smegmatis. The greatest increase in the sensitivity of test-culture of mycobacteria occurred with ampicillin, benzylpenicillin, cephazolin and ceftriaxone in combination with prodigiosin of $S$. marcescens. The presented combination of antibiotics and prodigiosin reduce the required concentration of the antibiotic and by amplifying the effect of compounds inhibiting cell wall synthesis, thereby giving lower FICI values. These data indicate the possibility of using prodigiosin as a promising candidate for the development of "accompaniment-preparations" for antibiotics for the additional therapy of infectious diseases caused by Mycobacterium spp. and can suspend the likelihood of developing resistance to antibiotics.

Keywords: natural pigment; antibiotics; outer membrane; peptidoglycan; combination activity.

\section{Introduction}

Mycobacterial infections are a group of multisystem infections caused by the members of the family Mycobacteriaceae. These organisms are characterized by their staining and identified as acid-fast bacilli. The most notable mycobacterial infections are those that are caused by Mycobacterium tuberculosis complex and M. leprae. Currently, tuberculosis (TB) is a major cause of morbidity and death in low- and middle-income countries. The emergence of drug-resistant strains has made the treatment of TB complex, costly, toxic, time-intensive, and less efficacious. The design of a treatment regimen for drug-resistant TB includes the administration of first-line drugs to which the strains remain susceptible together with second-line drugs. Too frequently, premature discontinuation of therapy occurs, leading to treatment failure and the emergence of $M$. tuberculosis strains with additional drug resistance (Cohen et al., 2014; Millard et al., 2015). Resistance to at least rifampicin and, isoniazid, also termed multidrug-resistant tuberculosis is on the rise. Though host genetic factors may play a role, insufficient or incomplete treatment is the most important determinant of the development of MDR tuberculosis (Pai et al., 2016; Nasiri et al., 2017).

Although M. tuberculosis is by far themost important mycobacterial species from a public health perspective, other species are being encountered with increasing frequency, and new species identified. Collectively, these organisms are referred to by a variety of terms, including anonymous or atypical mycobacteria, mycobacteria other than tuberculosis, and non-tuberculous (NTM) mycobacteria (McShane et al., 2015; Wu et al., 2015). Non-tuberculosis mycobacteria often cause extrapulmonary and pulmonary disease. Some non-tuberculous mycobacteria cause similar clinical and radiological characteristics with tuberculosis.
While most exposed and infected individuals never acquire NTM disease, some ostensibly immunocompetent persons will (Shahraki et al., 2015). Besides, some NTM pathogens also display acquired mutational drug resistance, which is the common mechanism of acquired drug resistance most physicians know because of their familiarity with TB (Cowman et al., 2015).

M. tuberculosis and other members of the M. tuberculosis complex use several strategies to resist the action of antimicrobial agents. First, the mycobacterial cell is surrounded by a specialized, highly hydrophobic cell wall that results in decreased permeability to many compounds. Active drug efflux systems and degrading or inactivating enzymes, and the genes that are associated with these functions have been found in M. tuberculosis. M. tuberculosis has been considered innately resistant to most $\beta$-lactam antibiotics that target peptidoglycan biosynthesis due to a highly active $\beta$-lactamase (BlaC) that efficiently inactivates many $\beta$-lactams and the fact that a large proportion of the cell wall peptidoglycan is cross-linked by non-classical L,d-transpeptidases, which are intrinsically impervious to these antibiotics (Bhat et al., 2017; Gygli et al., 2017). However, genetic studies have shown that resistance of $M$. tuberculosis to antimycobacterial drugs is the consequence of spontaneous mutations in genes that encode either the target of the drug, or enzymes that are involved in drug activation. Resistance-associated point mutations, deletions, or insertions have been described for all firstline drugs: isoniazid, rifampin, pyrazinamide, ethambutol, and streptomycin, and several second-line and newer drugs: ethionamide, fluoroquinolones, macrolides, nitroimidazopyrans (Cohen et al., 2014; Nasiri et al., 2017). Escalating the application of genomic techniques has expedited the identification of an increasing number of mutations associated with drug resistance in M. tuberculosis. Unfortunately, the preva- 
lence of bacillary resistance is becoming alarming in many parts of the world, with daunting scenarios of multidrug-resistant tuberculosis (MDR-TB), extensively drug-resistant tuberculosis (XDR-TB) and drug-resistant tuberculosis (TDR-TB), due to several resistance pathways, alongside some obscure ones (Hameed et al., 2018).

Though some of the therapeutic strategies have been employed to treat and eradicate the disease, the emergence of drug-resistant strains has attracted worldwide concern. There is a greater need of alternate chemotherapeutics because, in spite of the considerable amount of research in modern chemotherapy, the exploitation of new antimicrobial agents from natural resources is considered as an important task, particularly in developing countries where the threat of drug resistance in microorganism is greater. Prodigiosin is seen to be a promising agent in this regard (Pore et al., 2016). Different studies shown that prodigiosin inhibits the growth of a broad spectrum of gram-positive (Staphylococcus spp., Bacillus spp., etc.) as well as gram-negative (Escherichia coli, Salmonella enterica, etc.) bacteria (Danevčič et al., 2016). Nevertheless, the antimicrobial properties of prodigiosin have often been questioned, particularly because of the high concentrations required for it to be effective, as these exceed the levels causing toxicity in mammalian cells. For this reason, it has been studied in greater depth for its use in anticancer and immunosuppressive therapy than as an agent to fight infectious agents (Stankovic et al., 2014; Li et al., 2018). The interest in prodigiosin as a drug is demonstrated by the number of reports in the literature from the different fields concerned, as well as by the explorations of its complexation, and encapsulation, for both drug-delivery and to enhance its activity (Dozie-Nwachukwu et al., 2017). As of today, it is relevant to implement alternative methods of treatment of infections caused by different microorganisms and to develop combined approaches for the treatment of infectious diseases (Xu et al., 2018; PizzolatoCezar et al., 2019). The combined approach distinguished by the use of two or more therapies for the treatment of infectious diseases. Its advantage is the ability to overcome the individual limitations of each active substance (Mulani et al., 2019).

The objective of the study is to determine the synergy effect of prodigiosin in combinations with inhibitors of cell wall synthesis and review the cell wall as the target for pathogenic Mycobacterium spp.

\section{Materials and methods}

The experiment was performed on clinical Mycobacterium smegmatis isolate UCM B-917. The isolate obtained from D. K. Zabolotny Institute of Microbiology and Virology of the National Academy of Sciences (Kyiv, Ukraine). M. smegmatis UCM B-917 was initially grown for $24 \mathrm{~h}$ in nutrient broth in the presence of $0.05 \%$ Tween- 80 . The culture, grown in the above medium, served as an inoculum that was added to $250 \mathrm{~mL}$ of Sauton's fluid medium base (Shleeva et al., 2017; Baena et al., 2019), containing: glycerol $-60 \mathrm{~mL} / \mathrm{L}$; L-asparagine $-1.330 \mathrm{~g} / \mathrm{L}$; citric acid $-0.660 \mathrm{~g} / \mathrm{L}$; dipotassium hydrogen phosphate $0.177 \mathrm{~g} / \mathrm{L}$; magnesium sulphate 7-hydrate $-0.166 \mathrm{~g} / \mathrm{L}$; sodium dihydrogen phosphate $-0.056 \mathrm{~g} / \mathrm{L}$; sodium chloride $-0.035 \mathrm{~g} / \mathrm{L}$; ferric ammonium citrate $-0.0167 \mathrm{~g} / \mathrm{L}$. Sauton's medium does not contain albumin, and is an alternative medium for some applications. In this medium, $M$. smegmatis can be grown with shaking, which gives rise to planktonic growth, or in standing cultures as a surface pellicle. Glycerol added to the medium serves as a carbon source. Salts like ferric ammonium citrate and magnesium sulphate provide inorganic ions and nitrogen sources essential for the growth of Mycobacterium. Asparagine is added to promote the initiation of growth and increase the growth rate. Tween 80 is added to reduce the natural tendency of the cells to stick together in large clumps and it also protects against a substantial amount of contamination, since many other organisms are inhibited by the presence of this detergent. For solid medium, agar was added at $15 \mathrm{~g} / \mathrm{L}$ and Tween 80 was left out. The culture was incubated at $+37^{\circ} \mathrm{C}$ in aerobic conditions for 3-5 days. The resulting slurry compared to $0.5 \mathrm{McFar}-$ land turbidity standard and a final bacterial inoculum of $1-2 \times 10^{8}$ $\mathrm{CFU} / \mathrm{mL}$ was placed in each well.

As a pigment producer, we used the species $S$. marcescens, namely the pigment-forming strain, which was isolated in the laboratories of the
Department of Microbiology, Virology, and Immunology of Bogomolets National Medical University from the bentonite clays of Kurtsivskyi deposit (Crimea, Ukraine). Red colour pigment-producing bacteria with different morphology and individual colonies were picked up separately and purified by quadrant streaking in nutrient agar plates for the isolation of the bacterium $S$. marcescens. The pigmented colonies of bacteria were selectively isolated and transferred by the method of loop inoculum on nutrient agar surface of the following composition: glycerol - 10 $\mathrm{mL} / \mathrm{L}$; peptone $-10 \mathrm{~g} / \mathrm{L}$; yeast extract $-2 \mathrm{~g} / \mathrm{L} ; \mathrm{K}_{2} \mathrm{SO}_{4}-10 \mathrm{~g} / \mathrm{L} ; \mathrm{MgCl}_{2}$ $-1.4 \mathrm{~g} / \mathrm{L}$. The final $\mathrm{pH}$ of the medium was $7.2 \pm 0.2$. Then Petri dishes with inoculated strains of $S$. marcescens were incubated in a thermostat at $+28^{\circ} \mathrm{C}$ for $24-72 \mathrm{~h}$ in an inverted position for the screening of pigmentproducing strains. These obtained isolates were taken and identified by morphological and biochemical characterization using Bergey's manual of systematic bacteriology (Phatake et al., 2016; Shimathi et al., 2017). The identified bacterial isolate used for further studies.

The extraction of prodigiosin pigment from biomass of bacteria was carried out by double processing of biomass with $96 \%$ ethanol. The resulting preparation was dried in air and reextracted. The procedure was repeated several times before the release of insoluble admixtures. The resulting homogeneous solution was designated as a crude pigment complex or ethanol extract. The ethanol extract was evaporated dry in a drying oven at a temperature of $+45-50{ }^{\circ} \mathrm{C}$ and, the residue dissolved in chloroform $(10 \mathrm{~mL} / \mathrm{g}$ of precipitate). The resulting solution was mixed with an equal volume of a water-ethanol mixture (4:1) and emulsified on a magnetic stirrer for one hour at room temperature. A water-ethanol mixture containing water-soluble admixture was separated by a separating funnel. The procedure was repeated by increasing the volume content of ethanol by half. The drug was then redried in a vacuum oven and redissolved $(10 \mathrm{~mL} / \mathrm{g}$ of precipitate)in ethanol (Darshan et al., 2015).

The purity of prodigiosin isolated from the pigmented strain was determined by high-performance liquid chromatography (HPLC-MS) on the Agilent 1200 device (Agilent Technologies, USA) with diodematrix and mass-selective detectors. Detection was performed using a diode-matrix detector with 315 and $535 \mathrm{~nm}$ signal recording. The molecular weight of the compounds determined on a massive detector with ionization in positive and negative APCI mode.

Determination of the absorption spectrals of the isolated pigment was performed by UV/VIS spectrophotometry method (Pore et al., 2016; Chenqiang et al., 2019). Absorption spectra of the extract were tested by Portlab 512 spectrophotometer in the range 400-700 nm. Absorption of bacterial cells before extraction was noted at each stage. The concentration of pigment was calculated using the following equation (Hussan et al., 2015; Kimyon et al., 2016):

$$
\text { Concentration of prodigiosin }=\frac{\left[O D_{534}-\left(1.381 \times O D_{620}\right)\right] \times 1000}{O D_{620}}
$$

where $O D$ - optical density; $O D_{534}$ - represent pigment absorption; $O D_{620}$ - represent bacterial cells absorption; 1.381 - constant.

In this study we used antibiotic gradient synergy testing to evaluate antimicrobial combinations, which included: amoxiclav, ampicillin, benzylpenicillin, vancomycin, imipenem, cephazolin, ceftriaxone and as drugs for comparison - kanamycin and rifampicin in combination with prodigiosin pigment. Antibiotics were obtained from Pharmaceutical Darnitsa firm (Kyiv, Ukraine). All antibiotics were tested in combination with prodigiosin isolated from $S$. marcescens against clinical $M$. smegmatis isolate. Stock antibiotic solutions were prepared and dilutions made according to the CLSI (Clinical Laboratory Standardization Institute) method or manufacturer's recommendations (Wayne, 2015). All compounds, except rifampicin $(2.0 \mathrm{~mL}$ ethanol $+8.0 \mathrm{~mL}$ sterile distilled water) and pigment prodigiosin $(0.5 \mathrm{~mL}$ ethanol $+0.5 \mathrm{~mL}$ sterile distilled water) were dissolved in $10 \mathrm{~mL}$ of sterile distilled water to form their $(w / v)$ stock solution. Different concentrations of compounds were prepared and used during this study while the stock solutions were stored in a freezer at $-20^{\circ} \mathrm{C}$ (rifampicin and pigment prodigiosin at $+4{ }^{\circ} \mathrm{C}$ ) until use.

Disk diffusion tests performed by using Sauton's agar culture medium. The test was prepared by suspending colonies of M. smegmatis into Sauton's broth and adjusted to an optical density equal to 
$0.5 \mathrm{McF}$ arland standard. The organisms were evenly spread on the surface Sauton's agar plate using a cotton swab and allowed to dry for about $10 \mathrm{~min}$ before the disks were applied to the plate and duplicated plates performed. After the inoculation on the surface, Sauton's agar with a direct suspension of isolated colonies adjusted to turbidity standard, commercial antimicrobial disks were placed on the agar surface. Antibiotic discs (amoxiclav $10 \mu \mathrm{g}$, ampicillin $10 \mu \mathrm{g}$, benzylpenicillin 10 $\mu \mathrm{g}$, imipenem $10 \mu \mathrm{g}$, ceftriaxone $30 \mu \mathrm{g}$,cephazolin $30 \mu \mathrm{g}$, vancomycin $30 \mu \mathrm{g}$, kanamycin $30 \mu \mathrm{g}$ and rifampicin $5 \mu \mathrm{g}$ ) were from Hi Media Laboratories. After $72-96 \mathrm{~h}$ of incubation at $+37^{\circ} \mathrm{C}$, the results were interpreted as either sensitive, intermediate, or resistant according to the inhibitory zone diameters around the disks using CLSI breakpoints (Wayne, 2017; Wayne, 2018). The test was performed in duplicate and at two independent time points.

Limitations of the method include the difficulty in the interpretation of zones of inhibition, especially when the amount of drug in the disk is near the breakpoint of the drug. The result is a fine haze of growth within the zone of inhibition, which can be difficult to interpret. Just as the inoculum can affect the results of broth microdilution tests, too heavy an inoculum can also cause falsely resistant interpretations of the disk zones. In this case, as with other methods, careful attention to the turbidity of the organism suspension is critical (Alcaide et al., 2017).

The minimum inhibitory concentrations (MICs) for antibiotics alone and their combinations with pigment prodigiosin were determined in triplicate by the microbroth dilution method in Sauton's broth according to CLSI (Wayne, 2015). Different concentrations of each of the antibiotics ranging from 250 to $0.1 \mu \mathrm{g} / \mathrm{mL}$ were prepared, except for their combinations with pigment prodigiosin (antibiotics in combination with prodigiosin were in ratio 1:1). In some cases, it is possible to increase the dose of at least one of the compounds of the combination within the tolerable toxicity. One milliliter of each working antibiotic concentration was serially diluted in Saunton's broth. After the serial dilution, $100 \mu \mathrm{L}$ of each of the adjusted bacterial strains was dispensed into each tube containing each antibiotic or their combinations with prodigiosin pigment. The minimum inhibitory concentration (MIC) was expressed as the lowest concentrations which inhibited growth as judged by the lack of turbidity in the tube. As a control, a tube containing antibiotics alone and a tube containing inocula alone, in each rack, were incubated simultaneously along with other tubes containing inocula for MIC determination. The number of surviving cells was normalized to the number of cells counted on plates without antibiotic for each strain and expressed as the relative CFU (percent CFU).

The broth microdilution format is traditionally set up as 2-fold dilutions and the MIC does not represent as absolute value; for example, if the MIC is reported to be $32 \mu \mathrm{g} / \mathrm{mL}$, the "true" MIC would fall between the lowest concentration that inhibits the growth of the organism (the "reported" MIC) and the next-lowest concentration. For example, the "true" MIC for the above-described example would be between 32 and $16 \mu \mathrm{g} / \mathrm{mL}$. Thus, generally, the acceptable values are within one 2-fold dilution of the actual endpoint and standards that help to ensure quality results of the test have been proposed. A proper inoculum is critical for the determination of valid MICs. A too-heavy inoculum may result in falsely resistant MIC readings, whereas a too-light inoculum may give falsely susceptible MIC readings due to the inadequate growth of the organism in broth (Alcaide et al., 2017).

The tubes were incubated at $+37^{\circ} \mathrm{C}$ from 72 to $96 \mathrm{~h}$ and thereafter observed for growth or turbidity. Subsequently, a loopful of broth from each test tube not showing growth was inoculated into a nutrient agar plate. Thereafter, equal volumes of sterile nutrient broth were added into the test tube cultures and incubated further from 72 to $96 \mathrm{~h}$ at $+37^{\circ} \mathrm{C}$. Then, the tubes and agar plates were examined for growth or turbidity using the unaided eye. These experiments were repeated three times. The MBC was determined by adding $50 \mu \mathrm{L}$ of the suspensions from the wells which did not show any growth after incubation during MIC assays to $150 \mu \mathrm{L}$ of fresh broth. These suspensions were reincubated at $+37^{\circ} \mathrm{C}$ from 72 to $96 \mathrm{~h}$ The MBC was determined as the lowest concentration of extract which inhibited $100 \%$ growth of microorganisms (Phillips et al., 2017).
To evaluate the synergistic, additive, indifference or antagonistic effect of the combination, the fractional inhibitory concentration index (FICI) was calculated for each antibiotic combination (Singh et al., 2018; Xu et al., 2018) by computing the ratio of the MIC (MBC) of the combination divided by the MIC (MBC) of the antimicrobial alone for each agent, and then adding those two ratios together (Equation A). Briefly, FICI calculated as follows:

$$
\text { Equation } \mathrm{A}: \mathrm{FICI}\left[\frac{\mathrm{MIC}_{\mathrm{A}(+\mathrm{B})}}{\mathrm{MIC}_{\mathrm{A}}}\right]+\left[\frac{\mathrm{MIC}_{\mathrm{B}(+\mathrm{A})}}{\mathrm{MIC}_{\mathrm{B}}}\right]
$$

where, $\mathrm{MICA}(+\mathrm{B})=\mathrm{MIC}(\mathrm{MBC})$ of $\mathrm{A}$ in the presence of drug $\mathrm{B}$; $\mathrm{MICA}=\mathrm{MIC}(\mathrm{MBC})$ of drug A alone; $\mathrm{MICB}(+\mathrm{A})=\mathrm{MIC}(\mathrm{MBC})$ of $\mathrm{B}$ in the presence of drug $\mathrm{A} ; \mathrm{MICB}=\mathrm{MIC}(\mathrm{MBC})$ of drug $\mathrm{B}$ alone. The FICI data were interpreted using the following criteria: Synergy defined as a FICI of $\leq 0.5$; no interaction: $0.5-4.0$ (additive: 0.5-1.0; indifference: FICI 1.0-4.0) and antagonism by FICI of $>4.0$. Discrepant MIC results and those combinations with FICI $\leq 1$ were confirmed by performing an additional duplicate synergy test.

Results between the disk diffusion and the broth dilution can be compared and quantified using the CLSI cutoffs and categorical levels specified. Broth dilution report MICs $(\mu \mathrm{g} / \mathrm{mL})$ represents a continuous metric of per-unit concentrations. Disk diffusion provides an inhibition zone diameter, which is also a continuous metric but in $\mathrm{mm}$ of distance. A value of $\mathrm{P}<0.05$ was considered as statistically significant (Nuzzo, 2014; Amrhein et al., 2017; Colquhoun, 2017). One-way analysis of variance (ANOVA), variation and statistical processing of the obtained results were performed by using the specialized software Statistica 9.0 (StatSoft Inc., USA). Selective parameters presented in the work have the following designations: " $x$ " is the sample average; "SE" is the standard error of the average value.

\section{Results}

The impact of antimicrobial agents on the susceptibility of M. smegmatis in disk diffusion testing in conditions of a 5-day laboratory experiment is demonstrated in Figure 1. A comparative analysis of the susceptibility to antimicrobial agents in the disk diffusion test showed that M. smegmatis was the most susceptible to ceftriaxone and kanamycin, and moderately sensitive to vancomycin and prodigiosin. The susceptibility of the isolate to the antibacterial combinations with prodigiosin in disk diffusion testing was not similar to that of antimicrobial agents alone. In the presence of prodigiosin, antibacterial activities of all antibiotics were not enhanced.

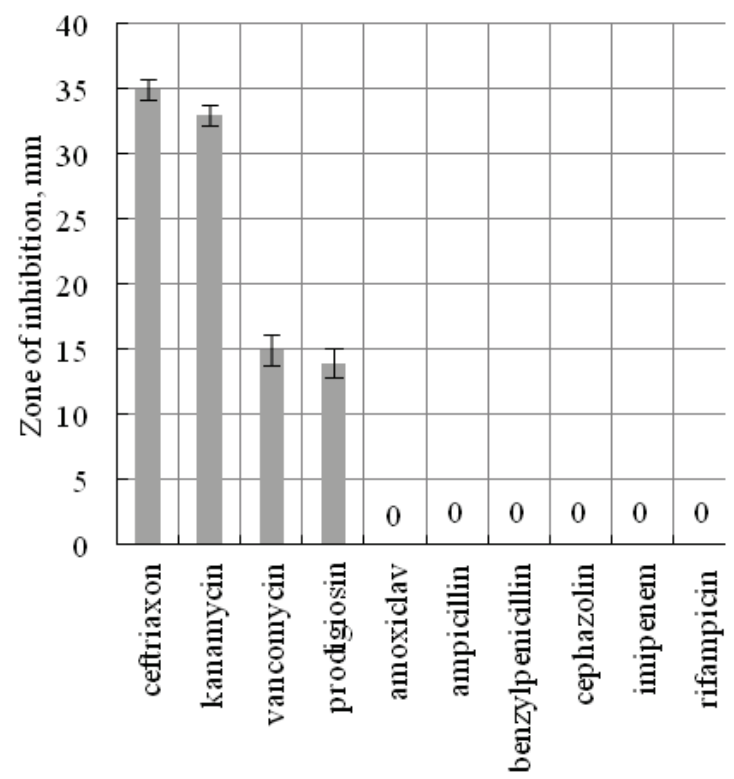

Fig. 1. Diameters of inhibition zones of growth $M$. smegmatis to the ten antimicrobial agents: amoxiclav, ampicillin, benzylpenicillin, cephazolin, imipenemand rifampicinno showed zone of inhibition

$(0 \mathrm{~mm})$; error bars represent means $\mathrm{x} \pm \mathrm{SE} ; *-\mathrm{P}<0.05$ 
The impact of antimicrobial agents on the susceptibility of $M$. smegmatis in the broth microdilution method in conditions of a 5-day laboratory experiment is demonstrated in Figure 2. A comparative analysis of the susceptibility to antimicrobial agents in the broth microdilution method showed that $M$. smegmatis was most sensitive to vancomycin, prodigiosin, kanamycin and rifampicin. On the contrary, the susceptibility of the isolate to amoxiclav, ampicillin, benzylpenicillin and imipenem did show inhibitory effect when making the maximum test concentration.

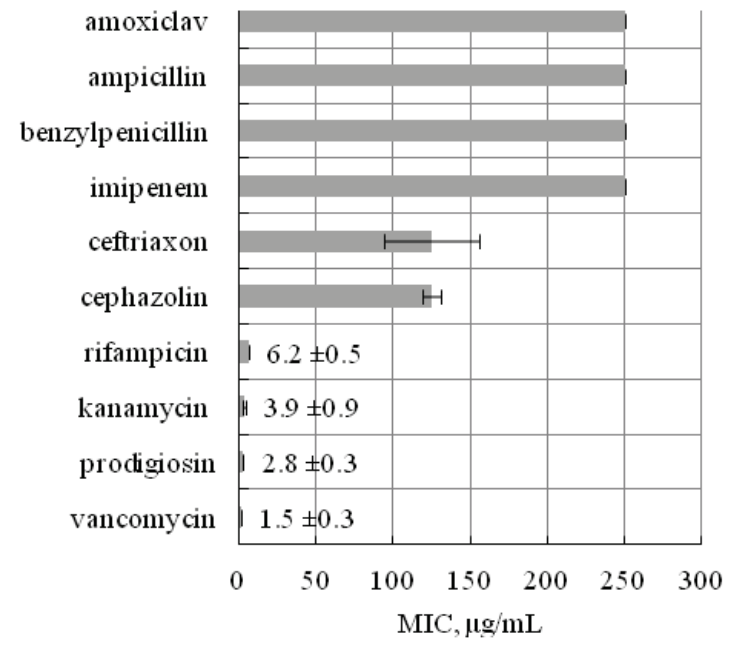

Fig. 2. Minimum inhibitory concentration (MIC) of the ten antimicrobial agents against $M$. smegmatis: amoxiclav, ampicillin, benzylpenicillin, and imipenem did not show inhibitory effect when making the maximum test concentration $(250 \mu \mathrm{g} / \mathrm{mL})$; error bars represent means $\mathrm{x} \pm \mathrm{SE}$; * $-\mathrm{P}<0.05$

The MICs of ten antimicrobial compounds and FICIs of nine antibiotics in combination with prodigiosin against clinical M. smegmatis isolate is shown in Table 1 . Combinatorial compounds sensitivity assays showed that $\mathrm{AM}, \mathrm{BZ}, \mathrm{CZ}, \mathrm{CF}$, and IM with prodigiosin (in ratio 1:1) presented remarcably synergistic activities against $M$. smegmatis with the FICIs from 0.076 to 0.374 . These values reflect a $>8$-fold reduction in the $\mathrm{MIC}$ for $\mathrm{AM}, \mathrm{BZ}, \mathrm{CZ}, \mathrm{CF}$ and $\mathrm{IM}$ a $>2$-fold reduction for prodigiosin (synergistic MIC) compared to the MIC of each compound alone.

\section{Table 1}

Broth dilution method MICs (range)

of indicated antibiotics alone and in combination with prodigiosin

\begin{tabular}{|c|c|c|c|c|c|c|}
\hline \multirow{2}{*}{ Antibiotics } & \multicolumn{4}{|c|}{ MIC $(\mu \mathrm{g} / \mathrm{mL})$, range } & \multirow{2}{*}{ ICI } & \multirow{2}{*}{ Interpretation } \\
\hline & $\mathrm{MIC}_{\mathrm{A}}$ & $\mathrm{MIC}_{\mathrm{A}(+\mathrm{B})}$ & $\mathrm{MIC}_{\mathrm{B}}$ & $\mathrm{MIC}_{\mathrm{B}}(+\mathrm{A})$ & & \\
\hline Prodigiosin & $5.6-2.8$ & - & - & - & - & - \\
\hline Benzylpenicillin & - & $1.4-0.7$ & 250 & 7.8 & $0.264-0$ & \\
\hline Amo & - & 5.6 & 250 & 31.2 & 0.529 & indinterenc \\
\hline $\mathrm{Am}$ & - & $1.4-0.7$ & 250 & 1.5 & 0.252 & synergy \\
\hline Vanc & - & $1.4-0.7$ & 1.5 & $0.7-0.3$ & 0.37 & indifference \\
\hline Imil & - & 1.4 & 250 & 0.6 & 0.251 & \\
\hline & - & $1.4-0.7$ & 125 & $15.6-7.8$ & & synergy \\
\hline & - & $0.3-0.1$ & $250-125$ & $7.8-3.9$ & 0.07 & synergy \\
\hline Rifat & - & 1.4 & 6.2 & 6.2 & $1.249-1.498$ & indifference \\
\hline Kanamycin & - & $0.7-0.3$ & $7.8-3.9$ & $7.8-3.9$ & $1.062-1.249$ & indifference \\
\hline
\end{tabular}

Note: $\mathrm{MIC}_{\mathrm{A}}$ - prodigiosin, $\mathrm{MIC}_{\mathrm{B}}$ - benzylpenicillin, amoxiclav, ampicillin, vancomycin, imipenem, cephazolin, ceftriaxone, rifampin, kanamycin, $\mathrm{MIC}_{\mathrm{A}}(+\mathrm{B})-$ prodigiosin (+benzylpenicillin, amoxiclav, ampicillin, vancomycin, imipenem, cephazolin, ceftriaxone, rifampin, kanamycin), $\mathrm{MIC}_{\mathrm{B}}\left(\mathrm{A}_{\mathrm{A}}\right)$ - benzylpenicillin, amoxiclav, ampicillin, vancomycin, imipenem, cephazolin, ceftriaxone, rifampin, kanamycin (+prodigiosin); benzylpenicillin, amoxiclav, ampicillin and imipenem did not show inhibitory effect when making the maximum test concentration $(250 \mu \mathrm{g} / \mathrm{mL})$; statistical analysis was done by one-way ANOVA; ${ }^{* * *}-\mathrm{P}<0.001$.

Data showed incompatible results of the disk diffusion test with the results of the broth dilution method for cephazolin, ceftriaxone, vancomycin, and prodigiosin and all of nine combinations with prodigiosin. On the contrary, ceftriaxone-sensitive isolate in the disk diffusion test had higher sensitivity than in the broth dilution method, but cephazolinsensitive isolate in the broth dilution method showed no zone of inhibition in the disk diffusion test. Significant differences between the results of disk diffusion and broth dilution methods were observed for ampicillin-, benzylpenicillin-, cephazolin- and imipenem-resistant isolate in combination with prodigiosin.

Other combinations with the FICIs from 0.529 to 1.498 showed no synergistic activity. The interaction of prodigiosin with the first-line drug rifampin and the second-line drug kanamycin was also analyzed in M. smegmatis. There was no synergy between prodigiosin and rifampicin or kanamycin, whereas a neutralistic profile was observed. The sensitivity of $M$. smegmatis to a set of five antibiotics with different structures and one cellular target analyzed in the presence of prodigiosin enhanced their activities. In these experiments, prodigiosin increased the potency (at least 8-fold) of ampicillin, benzylpenicillin, cephazolin, ceftriaxone, and imipenem against $M$. smegmatis. Importantly, the MIC of prodigiosinin combinations with antibiotics in vitro against $M$. smegmatis was 2- to 32-fold lower than alone.

Ampicillin, benzylpenicillin, vancomycin, cephazolin, ceftriaxone, and imipenemin combination with prodigiosin were tested in vitro to determine whether they were bacteriostatic or bactericidal against M. smegmatis. If a drug had bactericidal activity, culture growth would not resume after dilution into fresh medium. When the $\mathrm{MBC} / \mathrm{MIC}$ ratio for the individual drugs was determined, six antibiotics had bactericidal effect from 1.2 to $15.6 \mu \mathrm{g} / \mathrm{mL}$ in combination with 1.4 to $2.8 \mu \mathrm{g} / \mathrm{mL}$ of prodigiosin (Table 2).

Table 2

Broth dilution method MBCs of indicated antibiotics alone and in combination with prodigiosin

\begin{tabular}{lcccccc}
\hline \multirow{2}{*}{ Antibiotics } & \multicolumn{9}{c}{$\mathrm{MBC}(\mu \mathrm{g} / \mathrm{mL})$} & \multirow{2}{*}{ FCI } & \multirow{2}{*}{ Interpretation } \\
\cline { 2 - 6 } & $\mathrm{MBC}_{\mathrm{A}}$ & $\mathrm{MBC}_{\mathrm{A}}(+\mathrm{B})$ & $\mathrm{MBC}_{\mathrm{B}}$ & $\mathrm{MBC}_{\mathrm{B}}(+\mathrm{A})$ & & \\
\hline Prodigiosin & $>11.2$ & - & - & - & - & - \\
Benzylpenicillin & - & $>2.8$ & 250 & $>15.6$ & 0.312 & synergy \\
Amoxiclav & - & $>11.2$ & 250 & $>62.5$ & 1.250 & indifference \\
Ampicillin & - & $>2.8$ & 250 & $>3.1$ & 0.262 & synergy \\
Vancomycin & - & $>2.8$ & $>125$ & $>3.2$ & 0.299 & synergy \\
Imipenem & - & $>2.8$ & 250 & $>1.2$ & 0.254 & synergy \\
Cephazolin & - & $>2.8$ & $>250$ & $>31.2$ & 0.374 & synergy \\
Ceftriaxone & - & $>1.4$ & 250 & $>15.6$ & 0.186 & synergy \\
Rifampicin & - & $>2.8$ & $>12.4$ & $>12.4$ & 1.249 & indifference \\
Kanamycin & - & $>1.4$ & $>15.6$ & $>15.6$ & 1.124 & indifference \\
\hline
\end{tabular}

Note: see Table 1.

Interestingly, when prodigiosin was assayed in combination with a different fixed concentration of antibiotics, the $\mathrm{MBC}$ of prodigiosin was in the range 1.4-2.8 $\mu \mathrm{g} / \mathrm{mL}$, suggesting that the bactericidal character of prodigiosin was dominant in the combination. This effect was not observed when prodigiosin was assayed in combination with amoxiclav. These results revealed prodigiosin as a potential new bactericidal chemical entity for TB therapy.

The combinations of prodigiosin andampicillin, benzylpenicillin, cephazolin, ceftriaxone, or imipenem had a synergistic effect, without any antagonism and the mean FICI values were in a range from 0.076 to 0.374 . The combinations of prodigiosin and amoxiclav, kanamycin, or rifampicin FICI values were in a range from 0.529 to 1.498 representing had indifference effect. Interestingly, the combination of prodigiosin and vancomycin showed synergy only at higher concentrations of an antibiotic.

\section{Discussion}

For research on the effect of prodigiosin of bentonite strain of S. marcescens on the antimicrobial activity of inhibitors of cell wall synthesis and the susceptibility of Mycobacteria spp. to their joint action as test-culture, we selected M. smegmatis, as an alternative avirulent mycobacterial strain that is used as a surrogate for M. tuberculosis and has many common features with pathogenic mycobacteria. Routine in vitro screening for novel antimycobacterial compounds was facilitated by the availability of avirulent mycobacterial strains that are easy to 
grow. M. smegmatis is useful for the research analysis of other Mycobacteria species in laboratory experiments and could be useful at the initial phase of screening for new drugs (Wu \& Chan, 2016; Arthur et al., 2019). M. smegmatis is an aerobic, fast-growing with a fast doubling time, non-pathogenic mycobacterium that has the potential to adapt to microaerobiosis by changing from active growth to dormant or latent. It can be dormant in conditions of low oxygen concentrations and can survive for more than 650 days in the absence of carbon, nitrogen, and phosphorus states (Wu \& Gengenbacher, 2016; Trutneva et al., 2018). Furthermore, it is readily cultivatable in most synthetic or complex laboratory media, where it can form visible colonies in 3-5 days. This species shares more than 2000 homologous genes with M. tuberculosis and shares the same peculiar cell wall structure of M. tuberculosis and other mycobacterial species (Hiroyuki et al., 2016). M. smegmatis is particularly useful in understanding the cellular processes, including drug resistance, dormant state, fatty acid exchange, and gene expression regulation that are important to pathogenic mycobacteria like $M$. tuberculosis, M. leprae and M. avium subsp. paratuberculosis (Malhotra et al., 2017).

The first area of research, presented in the paper, was the evaluation of the susceptibility of mycobacteria to antimicrobial preparations, due to the impact of prodigiosin of S. marcescens and inhibitors of cell wall synthesis on M. smegmatis. In this research, we studied the suitability and reliability of disk diffusion to monitor the susceptibility of $M$. smegmatis isolate to inhibitors of cell wall synthesis. We have demonstrated that the disk diffusion testing had good correlations when compared with the broth dilution method (except cephalosporins). This finding is important because the disk diffusion test remains a common method used by many regional laboratories and hospitals for the detection of antimicrobial resistance. This observation is important because it suggests that disk diffusion remains appropriate as a routine method to detect isolates with non-susceptibility phenotype. The diffusion test appears appropriate for qualitative antibiotic susceptibility testing for clinicians to determine and choose the appropriate drug for treatment when no other testing alternatives are available. However, the disk diffusion test has additional limitations that should be considered. The test is labour-intensive and has similar limitations to other culture-based tests. Reading the disk diffusion test can be subjective, human errors can affect the outcome, and despite categorical classifications, it may have somewhat larger reproducibility variations than the agar dilution test. The reason for the variability of results between our study and those presented in other published studies for some of the antimicrobial combinations could be due to the difference in strains studied in different countries or differences in the methodologies used in these studies.

The second area of research is the combined use of prodigiosin with antibacterial drugs, presented in this publication, reproduced by the simultaneous effect of the prodigiosin pigment and inhibitors of cell wall synthesis on M. smegmatis. The results of the synergistic effect confirm and supplement the literature on the potentiation of conventional antibiotics and antimicrobial agents from natural resources. In summary, a set of seven antibiotics with different structures and one cellular target - cell wall, was analyzed in the presence of prodigiosin and identified six (except amoxiclav) as having activities synergistic with prodigiosin against M. smegmatis. The combinations of prodigiosin and ampicillin, benzylpenicillin, imipenem, cephazolin, or ceftriaxone had synergistic effects. The combinations of prodigiosin and amoxiclav, kanamycin, or rifampicin had an indifference effect. Prodigiosin works in combination and by amplifying the effect of compounds inhibiting cell wall synthesis, thereby giving lower FICI values, an important finding of our study.

A study of interactions of compounds showed that prodigiosin may be inactivating the different cellular targets, unlike inhibitors of cell wall synthesis, and act more efficiently together. Thus, the results of studies suggest that prodigiosin by hydrolysis of lipids, which predominate in the cell walls of mycobacteria leads to impaired permeability or embedded in the cell membrane of bacteria forms in its pores and passes antibiotics through it. Interestingly, prodigiosin enhanced the activity of inhibitors of cell wall synthesis, antibiotics that are not clinically effective in treating TB or NTM on its own. We demonstrated that prodigiosin could be used to enhance the activities of inhibitors of cell wall synthesis and maybe other non-clinically effective antibiotics against pathogenic or non-tuberculosis mycobacteria. The study has indicated that synergistic combinations of antimicrobial agents which are susceptible to pathogenic bacteria had a great potency to prevent resistance. The resultant synergy in the combination of prodigiosin and inhibitors of cell wall synthesis is a novel concept, as such combinations will have identical or different mechanisms of action, which may lead to new choices of therapeutic agents for the treatment, especially infections caused by multidrug-resistant microorganisms for which no effective therapy is available. Combinations of inhibitors of cell wall synthesis with prodigiosin may warrant further clinical investigation as treatments for tuberculosis and another the diseases associated with pathogenic Mycobacterium spp.

The third area of research is revisiting the cell wall as the target for treatment and the potential of targeting this essential structure to tackle Mycobacteria, especially M. tuberculosis. Most of the anti-TB drugs associated with cell wall biosynthesis inhibition lack the ability to reduce treatment duration of TB drug regimens. This is related to the fact that modification of cell wall targets mediated by specific enzymes or the accumulation of chromosomal mutations and degradation/modification of drugs by the production of antibiotic inactivating enzymes has rendered M. tuberculosis resistant to most classes of antimicrobials, and some bacteria can withstand the presence of the antibiotics by becoming dormant, i.e., being unable to replicate, as dormant bacteria do not actively synthesize the cell wall and are presumably not affected by the presence of inhibitors of the cell wall synthesis (Gygli et al., 2017; Nasiri et al., 2017). The limited number of new anti-mycobacterial agents approved for therapy and the wide variety of $M$. tuberculosis intrinsic and acquired drug resistance mechanisms to the available drugs have contributed to an increased effort to repurpose the use of antibiotics that are not commonly used in anti-TB therapy and to find suitable synergistic antibiotic combinations for effective treatment of life-risk TB (Diacon et al., 2016; Catalão et al., 2019). The results of the study made it possible to determine that the final result is influenced by the level of antimicrobial activity of the pigment used. The simultaneous use of the prodigiosin pigment of $S$. marcescens to enhance the susceptibility of mycobacteria to inhibitors of the cell wall synthesis, which have not been found clinically effective for the treatment against pathogenic Mycobacterium spp., are of great scientific importance.

\section{Conclusions}

The increase presented here in sensitivity of mycobacterium to antimicrobial drugs is due to the consistent influence of the metabolite of $S$. marcescens, and antibacterial preparation on M. smegmatis. The greatest increase in the sensitivity of test-culture of mycobacteria after the use of inhibitors of cell wall synthesis and prodigiosin was observed in relation to ceftriaxone and kanamycin, and moderately sensitive to vancomycin and prodigiosin. The absence of inhibition of the diameter of the growth was observed for the preparations of amoxiclav, kanamycin, and rifampicin. The susceptibility of the isolate to the antibacterial combinations with prodigiosin in disk diffusion testing did not show susceptibility similar to lone antimicrobial agents. The disk diffusion testing and broth dilution method produced comparable results of sensitivity for amoxiclav, ampicillin, benzylpenicillin, imipenem, and kanamycin, but neither methods showed comparable results for antibiotics in combination with prodigiosin. Evaluation of the effect of prodigiosin on the sensitivity of M. smegmatis in combination with different inhibitors of cell wall synthesis showed that the change in the sensitivity of the microbial cells of the pathogen was observed to varying degrees. The combinations of prodigiosin and ampicillin, benzylpenicillin, cephazolin, ceftriaxone, or imipenem had a synergistic effect, without any antagonism and the mean FICI values were in a range from 0.076 to 0.374 . The combinations of prodigiosin and amoxiclav, kanamycin, or rifampicin FICI values were in a range from 0.529 to 1.498 representing an indifference effect. Interestingly, the combination of prodigiosin and vancomycin $\mathrm{s}$ howed synergy only at higher concentrations of an antibiotic. The most active antibiotics among all the samples for enhancing the antimicrobialactivity of prodigiosin and increasing the sensitivityof $M$. smegmatis to their combined use are ceftriaxone and ampicillin. This makes prodigio- 
sin a promising candidate for the development of "accompaniment-preparations", for inhibitors of cell wall synthesis, which have not been considered as being useful drugs for the treatment of infectious diseases caused by Mycobacterium spp. and which can suspend the likelihood of developing resistance to other antibiotics.

\section{References}

Alcaide, F., Esteban, J., González-Martin, J., \& Palacios, J. J. (2017). Methods for determining the antimicrobial susceptibility of Mycobacteria. Enfermedades Infecciosaa in Microbiologia Clinica, 35(8), 529-535.

Amrhein, V., Korner-Nievergelt, F., \& Roth, T. (2017). The earth is flat $(\mathrm{P}>0.05)$ : Significance thresholds and the crisis of unreplicable research. The Journal of Life and Environmental Sciences, 5, e3544.

Arthur, P. K., Amarh, V., Cramer, P., Arkaifie, G. B., Blessie, E. J. S., Fuseini, M. S., Yeboah, R., Asare, L., \& Robertson, B. D. (2019). Characterization of two new multidrug-resistant strains of Mycobacterium smegmatis: Tools for routine in vitro screening of novel anti-mycobacterial agents. Antibiotics (Basel), 8(1), e4

Baena, A., Cabarcas, F., Alvarez-Eraso, K. L. F., Isaza, J. P., Alzate, J. F., \& Barrera, L. F. (2019). Differential determinants of virulence in two Mycobacterium tuberculosis Colombian clinical isolates of the LAM09 family. Virulence, 10(1), 695-710.

Bhat, Z. S., Rather, M. A., Maqbool, M., Lah, H. U., Yousuf, S. K., \& Ahmad, Z. (2017). Cell wall: A versatile fountain of drug targets in Mycobacterium tuberculosis. Biomedicine and Pharmacotherapy, 95, 1520-1534.

Catalão, M. J., Filipe, S. R., \& Pimentel, M. (2019). Revisiting anti-tuberculosis therapeutic strategies that target the peptidoglycan structure and synthesis. Frontiers in Microbiology, 10, 190.

Chenqiang, L., Xianbo, J., Yu, F., Longjun, C., Hui, Z., Rongbin, L., \& Jichen, C. (2019). Enhanced production of prodigiosin by Serratia marcescens FZSF02 in the form of pigment pellets. Electronic Journal of Biotechnology, 40, 58-64.

Cohen, K. A, Bishai, W. R., \& Pym, A. S. (2014). Molecular basis of drug resistance in Mycobacterium tuberculosis. Microbiology Spectrum, 2(3), e0036.

Colquhoun, D. (2017). The reproducibility of research and the misinterpretation of p-values. Royal Society Open Science, 4(12), 171085.

Cowman, S., Burns, K., Benson, S., Wilson, R., \& Loebinger, M. (2015). The antimicrobial susceptibility of non-tuberculous mycobacteria. Journal of Infection, 72(3), 324-331.

Danevčič, T., Vezjak, M. B., Zorec, M., \& Stopar, D. (2016). Prodigiosin - a multifaceted Escherichia coli antimicrobial agent. Public Library of Science One, 11(9), e0162412.

Darshan, N., \& Manonmani, H. K. (2015). Prodigiosin and its potential applications. The Journal of Food Science and Technology, 52(9), 5393-5407.

Diacon, A. H., van der Merwe, L., Barnard, M., von Groote-Bidlingmaier, F., Lange, C., García-Basteiro, A. L., Sevene, E., Ballell, L., \& Barros-Aguirre, D. (2016). $\beta$-lactams against tuberculosis - new trick for an old dog? The New England Journal of Medicine, 375(4), 393-394.

Dozie-Nwachukwu, S. O., Danyuo, Y., Obayemi, J. D., Odusanya, O. S., Malatesta, K., \& Soboyejo, W. O. (2017). Extraction and encapsulation of prodigiosin in chitosan microspheres for targeted drug delivery. Materials Science and Engineering: Materials for Biological Applications, 71, 268-278.

Gygli, S. M., Borrell, S., Trauner, A., \& Gagneux, S. (2017). Antimicrobial resistance in Mycobacterium tuberculosis: Mechanistic and evolutionary perspectives. FEMS Microbiology Reviews, 41(3), 354-373.

Hameed, H. M. A., Islam, M. M., Chhotaray, C., Wang, C., Liu, Y., Tan, Y., Li, X., Tan, S., Delorme, V., Yew, W. W., Liu J., \& Zhang, T. (2018). Molecular targets related drug resistance mechanisms in MDR-, XDR-, and TDRMycobacterium tuberculosis strains. Frontiers in Cellular and Infection Microbiology, 8, 114.

Hiroyuki, Y., Kinuyo, C., Akio, A., Yuriko, I., Yi, L., Akiko, T., \& Satoshi, M. (2016). Can M. smegmatis be used as a real alternative for M. tuberculosis? European Respiratory Journal, 48, 60 .

Hussan, H. T., \& Authaman, S. H. (2015). Antimicrobial and antiadhesive properties of prodigiosin extracted from Serratia marcescens against bacteria isolated from patients with leukemia. World Journal of Pharmaceutical Research, 4(12), 136-153.

Kimyon, Ö., Das, T., Ibugo, A. I., Kutty, S. K., Ho, K. K., Tebben, J., Kumar, N., \& Manefield, M. (2016). Serratia secondary metabolite prodigiosin inhibits Pseudomonas aeruginosa biofilm development by producing reactive oxygen species that damage biological molecules. Frontiers in Microbiology, 7, 972.

Li, D., Liu, J., Wang, X., Kong, D., Du, W., Li, H., Hse, C. Y., Shupe, T., \& Zhou, D., Zhao, K. (2018). Biological potential and mechanism of prodigiosin from
Serratia marcescens subsp. lawsoniana in human choriocarcinoma and prostate cancer cell lines. International Journal of Molecular Sciences, 19(11), e3465.

Malhotra, S., Vedithi, S. C., \& Blundell, T. L. (2017). Decoding the similarities and differences among mycobacterial species. PLOS Neglected Tropical Deseases, 11(8), e0005883.

McShane, P. J., \& Glassroth, J. (2015). Pulmonary disease due to non-tuberculous mycobacteria: Current state and new insights. Diseases of the Chest, 148(6), $1517-1527$.

Millard, J., Ugarte-Gil, C., \& Moore, D. A. (2015). Multidrug resistant tuberculosis. British Medical Journal, 350, h882.

Mulani, M., Kamble, E., Kumkar, S. N., Tawre, M. S., \& Pardesi, K. R. (2019). Emerging strategies to combat eskape pathogens in the era of antimicrobial resistance: A review. Frontiers in Microbiology, 10, 539.

Nasiri, M. J., Haeili, M., Ghazi, M., Goudarzi, H., Pormohammad, A., Fooladi, A. A., \& Feizabadi, M. M. (2017). New insights in to the intrinsic and acquired drug resistance mechanisms in mycobacteria. Frontiers in Microbiology, 8, 681.

Nuzzo, R. (2014). Scientific method: Statistical errors. Nature, 506(7487), 150-152.

Pai, M., Nicol, M. P., \& Boehme, C. C. (2016). Tuberculosis diagnostics: State of the art and future directions. Microbiol Spectrum, 4(5), e0019.

Phatake, Y. B., \& Dharmadhikari, S. M. (2016). Isolation and screening of prodigiosin production bacteria and characterization of produced pigment. International Journal of Security and Networks, 7(1), 202-209.

Phillips, D. J., Harrison, J., Richards, S. J., Mitchell, D. E., Tichauer, E., Hubbard, A. T. M., Guy, C., Hands-Portman, I., Fullam, E., \& Gibson, M. I. (2017). Evaluation of the antimicrobial activity of cationic polymers against Mycobacteria: Toward antitubercular macromolecules. Biomacromolecules, 18(5), 1592-1599.

Pizzolato-Cezar, L., Okuda-Shinagawa, N., \& Machini, M. (2019). Combinatory therapy antimicrobial peptide-antibiotic to minimize the ongoing rise of resistance. Frontiers in Microbiology, 10, 1703.

Pore, T. S., Ashwini, B. K., \& Naiem, H. N. (2016). Production, purification, identification of prodigiosin from Serratia sp. and its antimicrobial activity. Research Journal of Life Sciences, Bioinformatics, Pharmaceutical and Chemical Sciences, 1(6), 325.

Shahraki, A. H., Heidarieh, P., Bostanabad, S. Z., Khosravi, A. D., Hashemzadeh, M., Khandan, S., Biranvand, M., Schraufnagel, D. E., \& Mirsaeidi, M. (2015). "Multidrug-resistant tuberculosis" may be non-tuberculous mycobacteria. European Journal of Internal Medicine, 26(4), 279-284.

Shleeva, M. O., Trutneva, K. A., Demina, G. R., Zinin, A. I., Sorokoumova, G. M. Laptinskaya, P. K., Shumkova, E. S., \& Kaprelyants, A. S. (2017). Free trehalose accumulation in dormant Mycobacterium smegmatis cells and its breakdown in early resuscitation phase. Frontiers in Microbiology, 8, 524.

Singh, V., Bala, M., Bhargava, A., Kakran, M., \& Bhatnagar, R. (2018). In vitro efficacy of 21 dual antimicrobial combinations comprising novel and currently recommended combinations for treatment of drug resistant gonorrhoea in future era. PLoS One, 13(3), e0193678.

Srimathi, R., Priya, R., Nirmala, M., \& Malarvizhi, A. (2017). Isolation, identification, optimization of prodigiosin pigment produced by Serratia marcescens. International Journal of Latest Engineering and Management Research, 2(9), 11-21.

Stankovic, N., Senerovic, L., Ilic-Tomic, T., Vasiljevic, B., \& Nikodinovic-Runic, J. (2014). Properties and applications of undecylprodigiosin, and other bacterial prodigiosins. Applied Microbiology and Biotechnology, 98, 38413858.

Trutneva, K., Shleeva, M., Nikitushkin, V., Demina, G., \& Kaprelyants, A (2018). Protein composition of Mycobacterium smegmatis differs significantly between active cells and dormant cells with ovoid morphology. Frontiers in Microbiology, 9, 2083.

Wayne, P. A. (2015). Methods for dilution antimicrobial susceptibility tests for bacteria that grow aerobically. Clinical and Laboratory Standards Institute, Pennsylvania, USA.

Wayne, P. A. (2017). Performance standards for antimicrobial susceptibility testsing. Clinical and Laboratory Standards Institute, Pennsylvania, USA.

Wayne, P. A. (2018). Laboratory detection and identification of Mycobacteria. Clinical and Laboratory Standards Institute, Pennsylvania.

Wu, M. L., Chan, C. L., \& Dick, T. (2016). Rel is required for morphogenesis of resting cells in Mycobacterium smegmatis. Frontiers in Microbiology, 7, 1390.

Wu, M. L., Gengenbacher, M., \& Dick, T. (2016). Mild nutrient starvation triggers the development of a small-cell survival morphotype in Mycobacteria. Frontiers in Microbiology, 7, 947.

Wu, U.-I., \& Holland, S. M. (2015). Host susceptibility to non-tuberculous mycobacterial infections. The Lancet Infectious Diseases, 15(8), 968-980.

Xu, X., Xu, L., Yuan, G., Wang, Y., Qu, Y., \& Zhou, M. (2018). Synergistic combination of two antimicrobial agents closing each other's mutant selection windows to prevent antimicrobial resistance. Scientific Reports, 8, 7237. 